\title{
Исправления
}

Erratum

\author{
N.E. TOMOVA
}

\section{Erratum to: Natural Implication and Modus Ponens Principle}

\section{Tomova Natalya Evgenyevna}

Department of Logic, Institute of Philosophy, Russian Academy of Sciences. 14/5 Volkhonka St., Moscow, 119991, Russian Federation.

E-mail: natalya-tomova@yandex.ru

In the paper [1] some mistakes have occured and the main results are corrected herein.

1. The theorem 1 on page 140 is not correct for matrix

$\mathfrak{M}_{3}=<\{1,1 / 2,0\}, \rightarrow,\{1,1 / 2\}>$, where $\rightarrow$ is defined by truth-table:

\begin{tabular}{|c|ccc|}
\hline$\rightarrow$ & 1 & $1 / 2$ & 0 \\
\hline 1 & 1 & 1 & 0 \\
$1 / 2$ & 1 & 1 & $1 / 2$ \\
0 & 1 & 1 & 1 \\
\hline
\end{tabular}

In particular, lemmas 1 and 2, which are used in the proof of the theorem 1, don't hold for the above mentioned matrix ${ }^{1}$. More over, we can give a counter-example, which shows that the rule modus ponens is not tautologousness-preserving in matrix $\mathfrak{M}_{3}$. For example,

$\vDash_{\mathcal{M}_{3}} q \rightarrow((q \rightarrow(q \rightarrow r)) \rightarrow r)$ and

$\vDash_{\mathcal{M}_{3}}(q \rightarrow((q \rightarrow(q \rightarrow r)) \rightarrow r)) \rightarrow((p \rightarrow(q \rightarrow r)) \rightarrow((p \rightarrow q) \rightarrow(p \rightarrow r)))$, but $\nvdash_{\mathcal{M}_{3}}(p \rightarrow(q \rightarrow r)) \rightarrow((p \rightarrow q) \rightarrow(p \rightarrow r))$.

2. The assertion that the extended class of natural implications consists of 80 implications is not correct. So, there are only 18 natural implications (and respectively logical matrices) for which the rule modus ponens is tautologousness-preserving, but not truth-preserving.

2 implications when $D=\{1\}$ :

\footnotetext{
${ }^{1}$ The author is grateful to L. Devyatkin that he pointed out that fact.
} 


\begin{tabular}{|c|ccc|}
\hline$\rightarrow$ & 1 & $1 / 2$ & 0 \\
\hline 1 & 1 & 1 & 0 \\
$1 / 2$ & 1 & 1 & $a$ \\
0 & 1 & 1 & 1 \\
\hline
\end{tabular}

where $a \in\{0,1\}$.

16 implications when $D=\{1,1 / 2\}$ :

\begin{tabular}{|c|ccc|}
\hline$\rightarrow$ & 1 & $1 / 2$ & 0 \\
\hline 1 & 1 & $1 / 2$ & 0 \\
$1 / 2$ & $a$ & $a$ & $a$ \\
0 & 1 & 1 & 1 \\
\hline
\end{tabular}

\begin{tabular}{|c|ccc|}
\hline$\rightarrow$ & 1 & $1 / 2$ & 0 \\
\hline 1 & 1 & $1 / 2$ & 0 \\
$1 / 2$ & $a$ & $a$ & $1 / 2$ \\
0 & 1 & $1 / 2$ & 1 \\
\hline
\end{tabular}

\begin{tabular}{|c|ccc|}
\hline$\rightarrow$ & 1 & $1 / 2$ & 0 \\
\hline 1 & 1 & $b$ & 0 \\
$1 / 2$ & 1 & 1 & 1 \\
0 & 1 & 1 & 1 \\
\hline
\end{tabular}

\begin{tabular}{|c|ccc|}
\hline$\rightarrow$ & 1 & $1 / 2$ & 0 \\
\hline 1 & 1 & 0 & 0 \\
$1 / 2$ & $1 / 2$ & $1 / 2$ & $1 / 2$ \\
0 & 1 & 1 & 1 \\
\hline
\end{tabular}$\quad$\begin{tabular}{ccccc|}
$\rightarrow$ & 1 & $1 / 2$ & 0 \\
\hline 1 & 1 & 1 & 0 \\
$1 / 2$ & 1 & 1 & 1 \\
0 & 1 & $1 / 2$ & 1 \\
\hline
\end{tabular}

where $a \in\{1,1 / 2\}, b \in\{0,1\}$.

Thus, the extended class of natural implications consists of 48 implications (for 30 of which the rule modus ponens is truth-preserving).

\section{References}

[1] Tomova, N.E. "Natural Implication and Modus Ponens Principle", Logical Investigations, 2015, vol. 21, no 1, pp. 138-143. 